\title{
Riacho 3: Análise dos seus impactos ambientais, Caxias, Maranhão, Brasil
}

\section{Riacho 3: Analysis of their environmental impacts, Caxias, Maranhão, Brazil}

\author{
André Ricardo Ferreira da Silva Rocha 1, Helenice Silva Gomes 2, Ricardo Clayton Silva \\ Jansen 3, Tatiane Neves de Sousa 4, Domingas Hosanira Silva de Sousa 5, Gustavo da \\ Silva Gomes $\bullet 6$, Guilherme Sousa da Silva 7, Jailson da Costa Gaspar 8 , Maria de Fátima \\ Veras Araujo 99, Gonçalo Mendes da Conceição@10
}

${ }_{1}^{1}$ Mestranda do Programa de Pós-Graduação em Biodiversidade, Ambiente e Saúde/PPGBAS, Universidade Estadual do Maranhão/UEMA, Centro de Estudos Superiores de Caxias/CESC, Caxias, Maranhão, Brasil. E-mail: andrercrd06@gmail.com

${ }_{2}^{2}$ Mestranda do Programa de Pós-Graduação em Biodiversidade, Ambiente e Saúde/PPGBAS, Universidade Estadual do Maranhão/UEMA, Centro de Estudos Superiores de Caxias/CESC, Caxias, Maranhão, Brasil. E-mail: heleniceavlis14@gmail.com

${ }_{3}^{3}$ Mestranda do Programa de Pós-Graduação em Biodiversidade, Ambiente e Saúde/PPGBAS, Universidade Estadual do Maranhão/UEMA, Centro de Estudos Superiores de Caxias/CESC, Caxias, Maranhão, Brasil. E-mail: ricardojansen_20@hotmail.com

${ }^{4}$ Mestranda do Programa de Pós-Graduação em Biodiversidade, Ambiente e Saúde/PPGBAS, Universidade Estadual do Maranhão/UEMA, Centro de Estudos Superiores de Caxias/CESC, Caxias, Maranhão, Brasil. E-mail: tatiane.neves.bio@hotmail.com

${ }_{5}^{5}$ Mestranda do Programa de Pós-Graduação em Biodiversidade, Ambiente e Saúde/PPGBAS, Universidade Estadual do Maranhão/UEMA, Centro de Estudos Superiores de Caxias/CESC, Caxias, Maranhão, Brasil. E-mail: domingahosa@hotmail.com

${ }^{6}$ Bolsista de Iniciação Científica CNPq, Universidade Estadual do Maranhão/UEMA, Centro de Estudos Superiores de Caxias/CESC, Caxias, Maranhão, Brasil. E-mail: gustavogomes@aluno.uema.br

'Universidade Estadual do Maranhão/UEMA, Centro de Estudos Superiores de Caxias/CESC, Caxias, Maranhão, Brasil. E-mail: guilhermecx.cx@hotmail.com

${ }^{8}$ Universidade Estadual do Maranhão/UEMA, Centro de Estudos Superiores de Caxias/CESC, Caxias, Maranhão, Brasil. E-mail: Jailsoncosta18@hotmail.com

${ }^{9}$ Universidade Estadual do Piaui/UESPI, Teresina, Piauí, Brasil. E-mail: maria.fveras2018@gmail.com

10 Universidade Estadual do Maranhão/UEMA, Centro de Estudos Superiores de Caxias/CESC, Caxias, Maranhão, Brasil. E-mail: doutorgoncalo@gmail.com*Autor para correspondência

\section{Palavras-chave \\ Comunidades ribeirinhas \\ Degradação ambiental \\ Qualidade da água}

\section{Keywords}

Riverian Community Environmental Degradation Water Quality
Os sistemas aquáticos são fortemente impactados pelas atividades humanas, devido as diversas formas de utilização dos seus recursos. A identificação dos principais impactos ambientais é importante para caracterizar o nível de degradação e com isso sugerir medidas mitigadoras na área em degradação. 0 estudo teve como objetivo identificar as principais ações antrópicas e fatores ambientais que contribuem para a degradação do Riacho 3 no Município de Caxias/Maranhão. A coleta de dados procedeu-se em oito pontos, às margens do Riacho 3, no trecho do Bairro Pai Geraldo. Foram realizadas caracterizações quantitativas e qualitativas in loco dos parâmetros físico-químicos e fatores relevantes responsáveis pela degradação ambiental, respectivamente. Observou-se que, houveram variações significativas entre os parâmetros nos diversos pontos analisados, tal qual, diferentes níveis de intervenção antrópica. Infere-se que o Riacho 3 se encontra em processo de degradação ambiental, causado pelas ações dos recentes moradores locais. Dessa forma medidas do poder público municipal são necessárias para reduzir os impactos no Riacho 3 e preservar o manancial.

The aquatic systems are strongly impacted by human activities, due to the diverse ways of using their resources. The identification of the main environmental impacts is important to characterize the level of degradation and with that to suggest mitigating measures in the degraded area. This study aimed to identify the main anthropic actions and environmental factors that contribute to the degradation of Riacho 3 in the Municipality of Caxias, Maranhão. The data collection was carried out in eight points, on the banks of Riacho 3, in the section of the Pai Geraldo Neighborhood. Quantitative and qualitative characterizations were performed in loco of the physicalchemical parameters and relevant factors responsible for environmental degradation, respectively. It was observed that, there were significant variations between the parameters in the diverse analyzed points, as well, different levels of antropic intervention. It is inferred that Riacho 3 is in the process of environmental degradation, caused by the actions of the recent local residents. In this way measures of the municipal public power are necessary to reduce the impacts in Riacho 3 and to preserve the source. 


\section{(IMMES)}

\section{INTRODUÇÃO}

A água é um recurso de grande importância à sobrevivência humana e apresenta várias utilizações, porém com a expansão da população e das atividades industriais, a poluição das águas pode estar relacionada com a falta de saneamento básico e de gestão de resíduos sólidos (OLIVEIRA et al., 2017).

Segundo a Resolução do CONAMA N.ㅇ 001 de 23 de janeiro de 1986, impacto ambiental é definido como alterações nas propriedades físicas, químicas e biológicas do meio ambiente, oriundas de atividades antrópicas que, direta ou indiretamente, possam afetar a saúde, a segurança e o bem-estar da população; assim como, suas atividades sociais e econômicas; o ambiente e as condições de qualidade da natureza e recursos ambientais. Por isso, não só a identificação dos principais impactos ambientais é importante, mas, sobretudo, as medidas mitigadoras passíveis de serem aplicadas na área degradada (BRASIL, 1986; ALVES, 2012).

A demanda de água para a realização da maioria das atividades humanas, produzem uma grande quantidade de águas residuais, onde a medida que a demanda global aumenta por água, cresce a quantidade de efluentes produzidas e a carga total de poluição aumentam continuamente. Mais de $80 \%$ das águas residuais do mundo - e mais de $95 \%$ em alguns países subdesenvolvidos - são liberados para o meio ambiente sem tratamento (WWAP, 2017).

Os sistemas aquáticos continentais são fortemente impactados pelas atividades humanas, devido as diversas formas de utilização dos seus recursos. A diversificação dos usos torna os impactos ainda maiores e significativos, proporcionando uma difícil resolução dos problemas gerados, dados os mais diversos tipos de poluição a que estão submetidos (MATSUMURA-TUNDISI; TUNDISI, 2008).

De acordo com o Decreto 24.643 do Código das Águas (1934), as águas são classificadas como de uso comum, públicos e particulares, as normas sobre os usos das águas e de acesso foram estabelecidas destacando o valor de sua utilização para satisfazer as primeiras necessidades da vida, assegurando sua gratuidade e imprescritibilidade (BRASIL, 1934).

Ações como o uso impróprio dos solos, o desmatamento exacerbado e o uso irracional de fertilizantes, corretivos agrícolas e agrotóxicos, vêm acelerando o processo de degradação ambiental, sobre tudo em áreas de nascentes e ao longo de cursos d'água, fatores esses que agravam a preocupação com relação à conservação desse recurso. A qualidade da água para fins de consumo, é o aspecto mais questionado quando os recursos hídricos estão associados a resíduos urbanos, além de resíduos da atividade agroindustrial, de igual forma, construções de barragens, mudanças nos cursos d'água e a devastação das matas ciliares causam impactos ambientais irreversíveis, afetando diretamente a qualidade da água (LEMOS et al., 2010).

$\mathrm{O}$ estudo teve como objetivo identificar as principais ações antrópicas e fatores ambientais que contribuem para a degradação do Riacho 3, no trecho do Bairro Pai Geraldo, no Município de Caxias, Maranhão, Brasil, através de agentes causadores de impactos ambientais e por meio de parâmetros físico-químicos, a fim de sensibilizar as comunidades que vivem nas margens e utilizam o riacho.

\section{MATERIAL E MÉTODOS}

\section{Caracterização da área de estudo}

Caxias é um município situado na mesorregião leste do estado do Maranhão, na área do médio Itapecuru, com coordenadas geográficas 04 51' 43,63" Sul e 43 21' 50,27" Oeste (IBGE, 2010; ARAÚJO, 2012), com altitude de $66 \mathrm{~m}$, do nível do mar (BARROS, 2012). É limitado geograficamente pelas cidades de Codó, Aldeias Altas e Coelho Neto ao norte; e São João do Sóter, Governador Eugênio Barros, Parnarama, Matões, e Timon ao sul; ao leste pelo Estado do Piauí; a oeste pelos municípios de Buriti Bravo e Gonçalves Dias (GASPAR et al., 2016).

Exibe uma vegetação onde o Cerrado é predominante, com gradientes de cobertura da vegetação que variam desde cerrado ralo à cerradão, com proveminente de áreas com buritizais e babaçuais (CONCEIÇÃO; RUGGIERI, 2010; BARROS, 2012). Segundo Costa (2016), o clima do município é do tipo subúmido seco, e possui temperatura média anual de $28{ }^{\circ} \mathrm{C}$, umidade relativa do ar entre 70 e $73 \%$, e precipitação pluviométrica variante entre 1600 a $2000 \mathrm{~mm}$ por ano, definindo duas estações, a chuvosa, correspondentes aos meses de dezembro a junho, e a outra seca, de julho a novembro. As características predominantes dos solos são do tipo latossolos vermelho - alaranjado podozólico vermelho - amarelado, areias e solos aluviais (BARROS, 2012).

O Rio Itapecuru e seus afluentes irrigam a cidade de Caxias, Maranhão (BARROS, 2012), dentre estes destacam-se para a região o Riacho São José e o Riacho 3 (Figura 1), com trechos nos bairros Pai Geraldo, Baixinha e Galeana. Segundo Silva (2011), este riacho, genuinamente maranhense, possui grande importância para o desenvolvimento econômico e povoamento do município. 
André Ricardo Ferreira da Silva Rocha, Helenice Silva Gomes, Ricardo Clayton Silva Jansen, Tatiane Neves de Sousa, Domingas Hosanira Silva de Sousa, Gustavo da Silva Gomes, Guilherme Sousa da Silva, Jailson da Costa Gaspar, Maria de Fátima Veras Araujo, Gonçalo Mendes da Conceição

Figura 1. Localização geográfica do Riacho 3 no município de Caxias, Maranhão, Brasil.

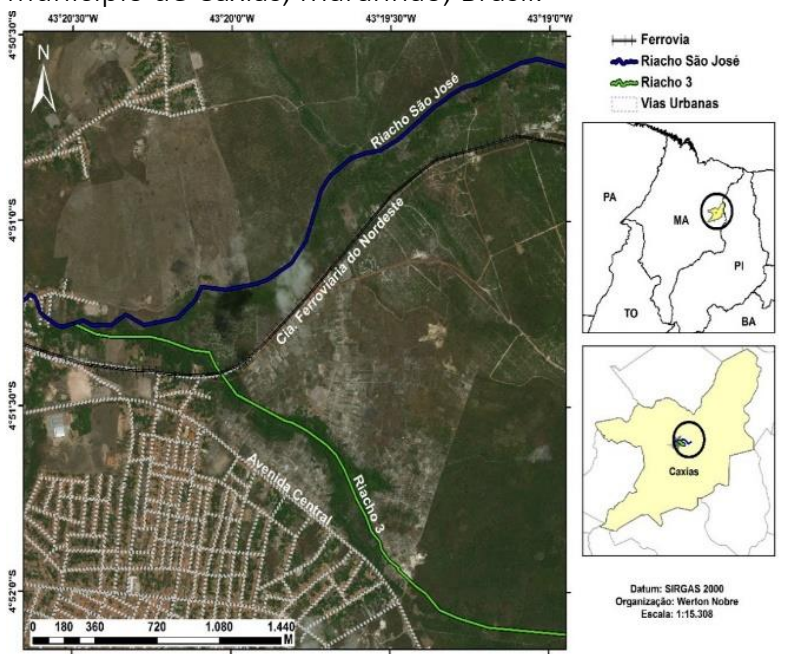

Fonte: O Autor (2017). descritos foram: Uso Doméstico do Riacho, Resíduos sólidos objetáveis, Presença ou Ausência de Mata Ciliar, Cor/Odor, Matéria orgânica em decomposição Bioindicadores. A vantagem desse método, além de ser realizada em curto espaço de tempo, proporciona menores gastos e é facilmente compreensível pelo público em geral.

\section{RESULTADOS E DISCUSSÃO}

Tabela 1. Resumo da metodologia utilizada nos parâmetros físico-químico.

\begin{tabular}{cc}
\hline Parâmetro & Metodologia \\
\hline Temperatura & Princípio de célula de Clark \\
\hline $\mathrm{pH}$ & Eletrométrico \\
\hline $\begin{array}{c}\text { Oxigênio } \\
\text { dissolvido }\end{array}$ & $\begin{array}{c}\text { Princípio de célula de Clark/ } \\
\text { método eletrométrico }\end{array}$ \\
\hline
\end{tabular}

O Riacho 3 em Caxias, pode ser agrupado como pertencente a Classe 3 - caracterizado pelo abastecimento para consumo humano, após tratamento convencional ou avançado; à irrigação de culturas arbóreas, cerealíferas e forrageiras; à pesca amadora; à recreação de contato secundário; à dessedentação de animais. Abaixo segue a Tabela 2, com os principais parâmetros físico-químicos e biológicos, analisados no Riacho 3.

\section{Temperatura do Ambiente e da Água}

Foi constatado durante as verificações, variações significativas de temperatura do ambiente com elevação máxima de $33,6^{\circ} \mathrm{C}(\mathrm{P} 1)$, mínima de $25,5^{\circ} \mathrm{C}$ (P8) e média de $29,7{ }^{\circ} \mathrm{C}$. Na verificação da água, a temperatura variou de 25,2 ${ }^{0} \mathrm{C}(\mathrm{P} 8)$ à uma máxima de $30,1^{\circ} \mathrm{C}(\mathrm{P} 3)$ e média de $23,3^{\circ} \mathrm{C}$. Os mais baixos picos de temperatura no $\mathrm{P} 8$, se explicam pela significativa distância entre este local e as moradias do Bairro Pai Geraldo, além da abundante vegetação às margens desse trecho. $\mathrm{O}$ valor máximo encontrado foi em P3, mas estudos apontam que esses resultados não agridem a biota local. De acordo com Von Sperling (2007), a temperatura dos ambientes locais variam de 22 a $30^{\circ} \mathrm{C}$.

Diversos autores, relacionam a temperatura com a oxigenação da água, pois o oxigênio é mais dissolvido na água quando este meio apresenta menor temperatura, já no caso de temperaturas elevadas ocorre a evaporação desse gás, sendo assim, menor a quantidade de oxigênio dissolvido (USA FEDERATION, 2012). 


\section{Potencial Hidrogeniônico $(\mathrm{pH})$}

$\mathrm{O}$ estudo evidenciou um $\mathrm{pH}$ considerado ácido que variou de 4,8 (P3, P4 e P8) a 5,6 (P5), com uma média de 5,1, esses valores não condizem com a Resolução do CONAMA $357 / 2005$, uma vez que os parâmetros aceitáveis para corpos hídricos de classe 3 estão entre 6 a 9 (Tabela 2).

$\mathrm{Na}$ área foi notória a presença de uma vegetação mais densa, de resíduos sólidos e muita matéria orgânica, em que este último indica ser um dos motivos para os valores do $\mathrm{pH}$ obtidos, sobretudo nos P4 e P8. Segundo Esteves (1998), isso deve-se ao fato de que este parâmetro é extremamente influenciado pela quantidade de matéria orgânica em decomposição, sendo assim, quanto mais matéria a ser decomposta, menor será $\mathrm{pH}$, pois durante o processo de decomposição desses materiais muitos ácidos são produzidos.

\section{Oxigênio Dissolvido na Água (OD)}

No que diz respeito ao parâmetro físico-químico de Oxigênio Dissolvido (OD), todo o trecho do riacho apresentou resultados dentro dos limites aceitáveis determinados na resolução do CONAMA 357/2005, com menor valor em P8 com valor de $6,9 \mathrm{mg} / \mathrm{L}$ e em P1 com maior valor $9,2 \mathrm{mg} / \mathrm{L}$. A média de OD levando em

Figura 2. Locais de banho, lazer e lavagem de vestimentas do Riacho 3 no Bairro Pai Geraldo em Caxias, Maranhão, Brasil. (A) Local de Banho; (B) Local de lavar roupa
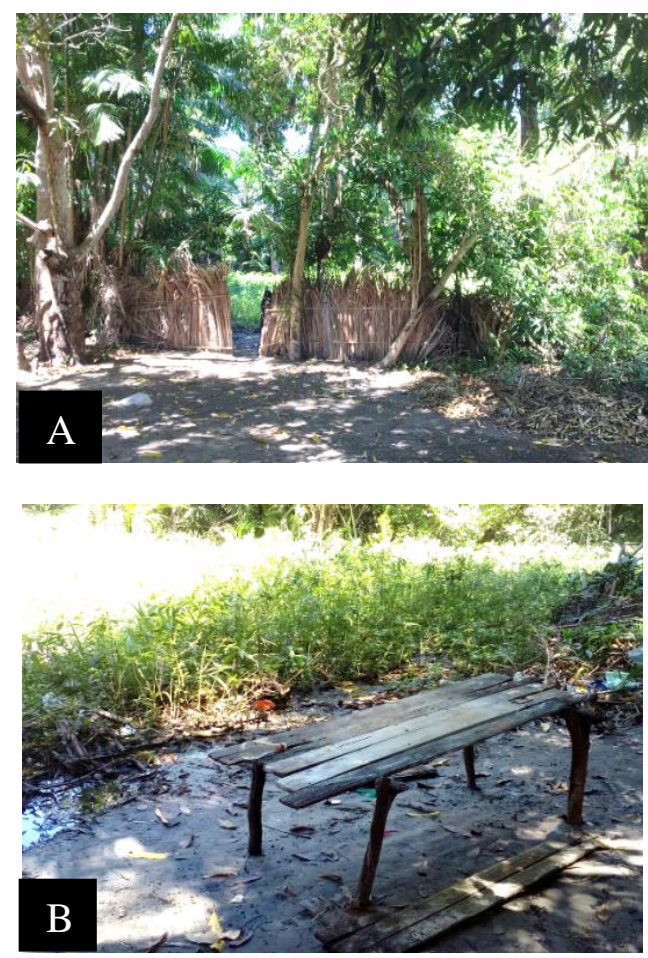

Fonte: O Autor (2017) consideração todos os pontos foi de $8,1 \mathrm{mg} / \mathrm{L}$ (Tabela 2).

Isso se deve pelo acúmulo de matéria orgânica proveniente dos lançamentos despejados ao longo do curso d'água que não foram neutralizados durante o processo de autodepuração, devido a alguns fatores como a baixa velocidade das águas no entorno dos pontos de coleta, podendo dificultar a autodepuração da matéria orgânica despejada no Riacho 3 (NOGUEIRA et al., 2015). Esses processos presentes principalmente em ambientes lênticos a viabilidade de distribuição do oxigênio é bem menor do que em ambientes lóticos. Segundo Esteves (1998), a difusão do oxigênio é ocasionada pelo o transporte de massas d'água sendo superior a difusão molecular. Outro ponto considerável para menor quantidade de OD pode ser em decorrência da atividade microbiana e zooplâncton no consumo de oxigênio e liberação de $\mathrm{CO}^{2}$ no meio aquático fenômeno observado em locais como P8 com águas escuras e $\mathrm{pH}$ baixo.

\section{Cor e Odor}

Outro parâmetro analisado para a determinação da qualidade da água, são os aspectos de cor e odor. Observouse que a coloração da água não era transparente em todos os pontos e quanto ao Odor não foi perceptível exalação que indicam poluição. Em consideração com a legislação do Conselho Nacional de Meio Ambiente CONAMA 357/2005 para o parâmetro cor todos os pontos ficariam fora de especificação. Mas neste caso não podemos afirmar que as alterações visualizadas de cor são causadas em decorrências de ações antrópicas, pois não foi evidenciado a presença de substâncias que levam a alterações, mas sim como um processo natural de decomposição de matéria orgânica vegetal com presença de substâncias responsável por esse processo de alteração de cor.

A presença de tanino, proveniente dos dominantes buritizais acarretam essa transparência. O tanino, um polieletrólito, quando combinado com sais metálicos possui o caráter de coagulante, que remove partículas sob as dimensões coloidais e suspensas por sedimentação e filtração, além da clarificação, a presença de taninos reduz diversos patógenos (SILVA, 1999; MARTINI; MORUZZI, 2013; SOUSA, 2015). Além de que locais como esses geralmente existe uma grande demanda de material residual de vegetação, como: folhas, gravetos, troncos e dentre outros que aumentam a produção de ácido fúlvicos e húmicos, principais responsáveis por alterações na tonalidade de cor da água. 
André Ricardo Ferreira da Silva Rocha, Helenice Silva Gomes, Ricardo Clayton Silva Jansen, Tatiane Neves de Sousa, Domingas Hosanira Silva de Sousa, Gustavo da Silva Gomes, Guilherme Sousa da Silva, Jailson da Costa Gaspar, Maria de Fátima Veras Araujo, Gonçalo Mendes da Conceição

Além dos parâmetros físico-químicos, foram observados diversos fatores relacionados ao grau de degradação ambiental, estes, contribuem para uma melhor análise geral, assim como possibilitam uma perspectiva futura do estado de conservação do Riacho 3, no trecho do bairro Pai Geraldo em Caxias, Maranhão, Brasil.

\section{Uso Doméstico do Riacho}

Esse parâmetro foi realizado através de conversas informais com os moradores, assim como constatações observadas no próprio local, o Riacho 3, é utilizado pelas comunidades ribeirinhas, para o banho, lazer, prática inicial de piscicultura, lavagem de vestimentas, dentre outras atividades (Figura 2). Atividades semelhantes foram observadas no Riacho Ouro, no município de Caxias, Maranhão (GASPAR et al., 2016), segundo este autor, essa intervenção da comunidade com o riacho, pode favorecer o processo de degradação do ambiente local.

\section{Resíduos Sólidos Objetáveis}

Verificou-se no riacho a presença de resíduos sólidos como pneus, sacolas descartáveis, garrafas pet e de vidro, roupas, dentre outros resíduos (Figura 3). Esses resíduos foram encontrados em locais de maior acesso de moradores da região nos pontos determinados P5, P6, P7 e P8. Conforme Araújo e Oliva Júnior (2012) a poluição em riacho ocorre principalmente pela ação antrópica próximo ao curso d'água e os vários tipos de resíduos sólidos depositados nas margens do riacho contribui para a degradação local.

\section{Presença ou Ausência de Mata Ciliar}

Outro parâmetro analisado, foi o cenário da vegetação no decorrer da extensão dos pontos analisados no

Figura 3. Resíduos sólidos depositados no Riacho 3 no Bairro Pai Geraldo em Caxias, Maranhão, Brasil. (A) Garrafas e dejetos sólidos; (B) Plásticos depositados na margem do riacho.
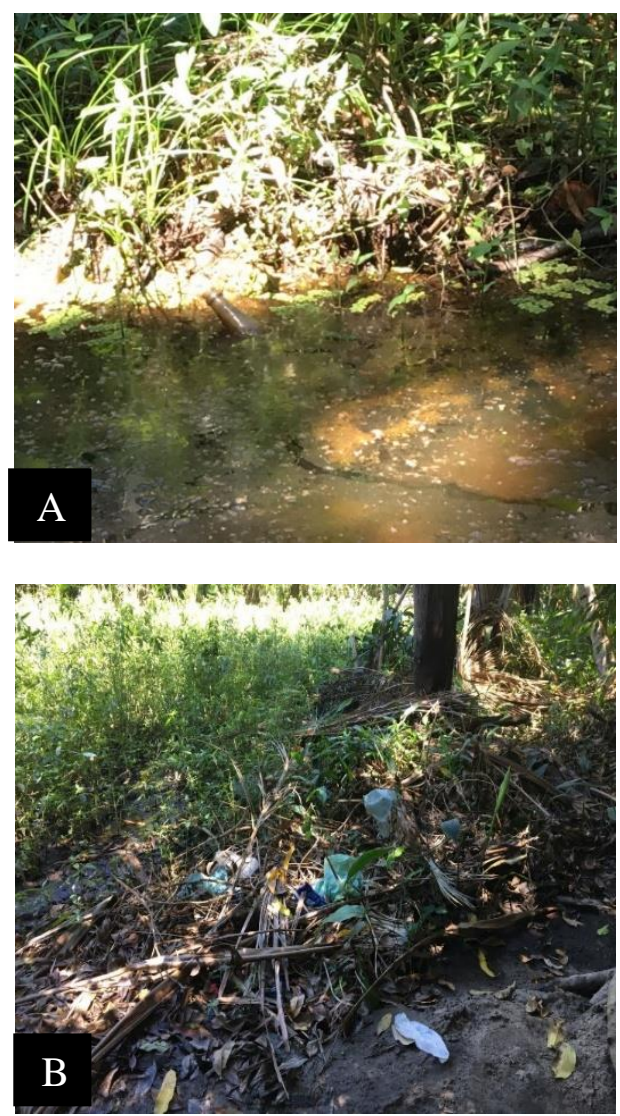

Fonte: O Autor (2017)

Tabela 2. Parâmetros físico-químicos e biológicos dos oito pontos analisados no Riacho 3, Bairro Pai Geraldo, Caxias, Maranhão, Brasil.

\begin{tabular}{|c|c|c|c|c|c|c|c|c|c|c|}
\hline Parâmetros & P1 & $\mathbf{P 2}$ & P3 & P4 & P5 & P6 & P7 & P8 & $\begin{array}{l}\text { CONAMA } \\
357 / 2005 \\
\end{array}$ & $\begin{array}{c}\text { Outras } \\
\text { referências }\end{array}$ \\
\hline Temp. do ar & 33,6 & 33,4 & 28,7 & 29 & 29 & 28,6 & 31,2 & 25,5 & & \\
\hline Temp. da água & 25,8 & 25,7 & 29,4 & 26,1 & 26,1 & 25,8 & 26,8 & 25,2 & & \\
\hline $\mathrm{Ph}$ & 5,4 & 5,3 & 4,8 & 4,8 & 5,6 & 5,2 & 5 & 4,8 & 6 a 9 & \\
\hline OD & 9,2 & 8,7 & 8,4 & 8,5 & 7,4 & 8,3 & 7,8 & 6,9 & $\geq 4 \mathrm{mg} / \mathrm{L}$ & \\
\hline Cor & $\mathrm{TC}$ & TE & $\mathrm{TE}$ & TE & TA & TC & TE & $\mathrm{TE}$ & Ausente & \\
\hline Odor & A & $A$ & $A$ & $A$ & $A$ & $A$ & $A$ & $A$ & Ausente & \\
\hline Bioindicadores & $\mathrm{P}$ & $\mathrm{P}$ & $\mathrm{P}$ & $\mathrm{P}$ & $\mathrm{P}$ & $\mathrm{P}$ & $\mathrm{P}$ & $\mathrm{P}$ & & \\
\hline Resíduos sólidos & $A$ & A & $A$ & A & $\mathrm{P}$ & $\mathrm{P}$ & $\mathrm{P}$ & $\mathrm{P}$ & Ausente & \\
\hline Mata ciliar & $\mathrm{P}$ & $\mathrm{P}$ & $\mathrm{P}$ & $\mathrm{P}$ & $A$ & $A$ & $A$ & $\mathrm{P}$ & & Presença \\
\hline Uso doméstico & $\mathrm{P}$ & $\mathrm{P}$ & $\mathrm{P}$ & $\mathrm{P}$ & $\mathrm{P}$ & $\mathrm{P}$ & $\mathrm{P}$ & $\mathrm{P}$ & & \\
\hline Mat.Org. decom & $\mathrm{P}$ & $\mathrm{P}$ & $\mathrm{P}$ & $\mathrm{P}$ & $\mathrm{P}$ & $\mathrm{P}$ & $\mathrm{P}$ & $\mathrm{P}$ & & \\
\hline $\begin{array}{l}\text { pH: Potencial Hidrog } \\
\text { OD: Oxigênio Dissolv }\end{array}$ & & $\begin{array}{l}\text { TC: Tra } \\
\text { TE: Tra }\end{array}$ & $\begin{array}{l}\text { sparer } \\
\text { sparer }\end{array}$ & e clara & & $\begin{array}{l}\text { P: Al } \\
\text { onte: }\end{array}$ & $\begin{array}{l}\text { cia ou } \\
\text { utor (2 }\end{array}$ & $\begin{array}{l}\text { esença } \\
\text { 7) }\end{array}$ & & \\
\hline
\end{tabular}


Figura 4. Mata ciliar do Riacho 3 no Bairro Pai Geraldo em Caxias, Maranhão, Brasil. A e B. Áreas desmatadas de mata ciliar; C e D. Áreas preservadas de mata ciliar.
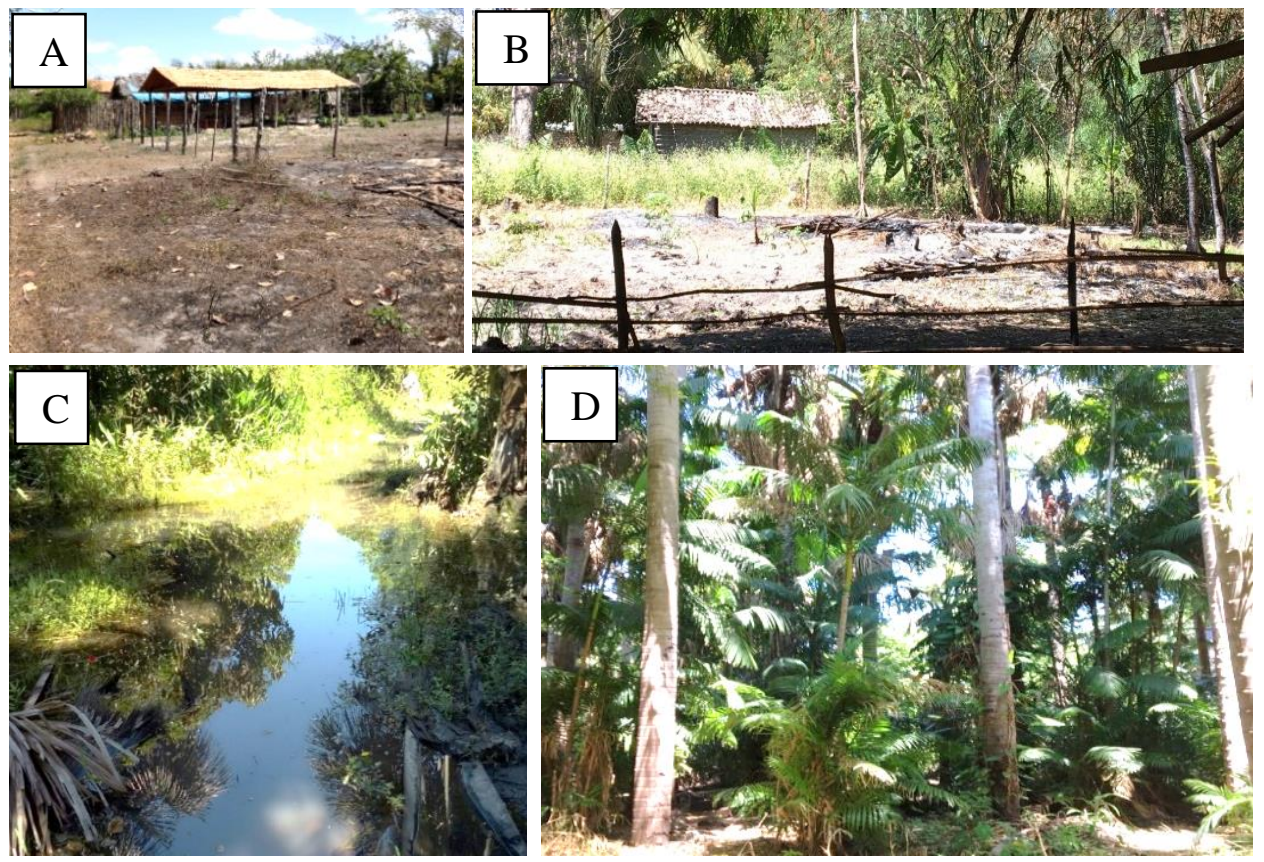

Fonte: O Autor (2017)

riacho. A presença ou ausência de matar ciliar não é um parâmetro relevante de qualidade de acordo com a resolução do CONAMA 357/2005 (BRASIL, 2005). No entanto, os resultados de mata ciliar foram comparados de acordo com o código florestal brasileiro Lei $\mathrm{n}^{\circ} 4.771$, de 15 de setembro de 1965 que determina áreas de proteção permanente situados ao longo de cursos d'águas (BRASIL, 1965).

De acordo com a lei $n^{\circ} 4.771 / 65$ somente os pontos P5, P6 e P7 não atenderam o código florestal brasileiro, pois apresentaram áreas desmatadas, queimadas (Figura 4) ou com quantidades de vegetação insuficiente para a garantia da conservação do leito do riacho, diferentemente dos outros pontos que foi possível verificar uma mata ciliar significativamente preservada. Esses locais estão situados com maior distância em relação aos outros das residências (Figura 4). De acordo com Cardoso e Novaes (2013) estes impactos ambientais, causados por ações antrópicas, têm levado a uma degradação da qualidade da água em diversos corpos hídricos, dificultando a utilização de tal recurso para a satisfação até mesmo das necessidades básicas humanas.

A vegetação no entorno do Riacho 3 é composta de buritizais que apresentam abundante concentrações da palmeira Mauritia flexuosa Mart. em meio a grupos densos de espécies arbustivas e herbáceas, que são o elemento dominante no estrato em que se inserem (SILVA, 2009; RIBEIRO, 2010).
Figura 5. Matéria orgânica em decomposição do Riacho 3 no Bairro Pai Geraldo em Caxias, Maranhão, Brasil. Áreas com muitas folhas, galhos e material vegetal em decomposição.
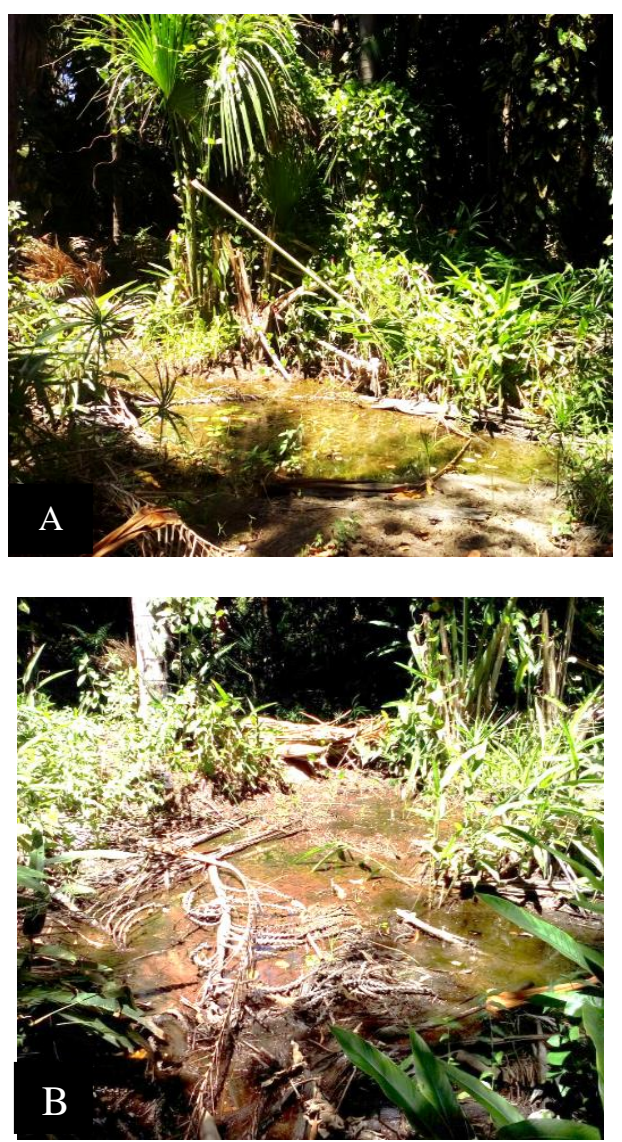

Fonte: O Autor (2017) 
André Ricardo Ferreira da Silva Rocha, Helenice Silva Gomes, Ricardo Clayton Silva Jansen, Tatiane Neves de Sousa, Domingas Hosanira Silva de Sousa, Gustavo da Silva Gomes, Guilherme Sousa da Silva, Jailson da Costa Gaspar, Maria de Fátima Veras Araujo, Gonçalo Mendes da Conceição

\section{Matéria Orgânica em Decomposição}

Outro parâmetro verificado foi a abundante quantidade de matéria orgânica em decomposição nas margens do riacho, como folhas, frutos, raízes e caule, principalmente de palmeiras e especificamente de buritizais (Figura 5). Esses restos vegetais influenciam no potencial hidrogeniônico $(\mathrm{pH})$ e no oxigênio dissolvido (OD) presente nas águas (ESTEVES, 1998).

Figura 6. Presença de bioindicadores no Riacho 3 no Bairro Pai Geraldo em Caxias, Maranhão, Brasil. (A) Espécie vegetal Ricinus communis L. (mamona); (Abaixo) Libélula.
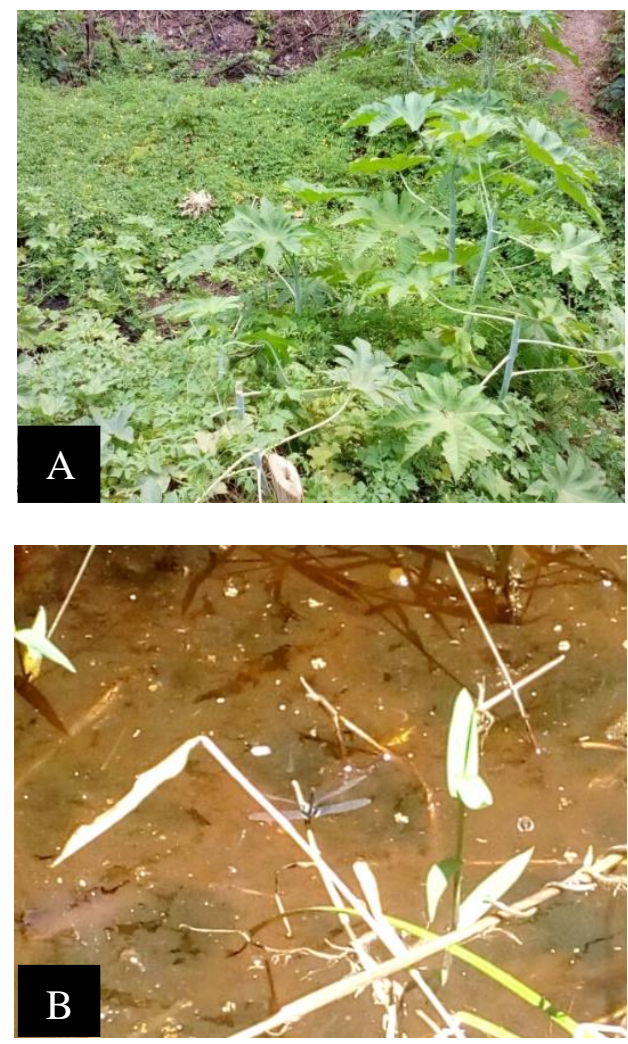

Fonte: O Autor (2017)

\section{Presença de Bioindicadores}

Com relação aos indicadores biológicos constatou-se a presença de espécies vegetais como Ricinus communis L. (mamona), Mauritia flexuosa L. f. (buriti), Clitoria fairchildiana (fava) e de espécies animais como Libélulas (Ordem Odonata), Borboletas (Ordem Lepidoptera) e alevinos (Figura 6).

Com relação as comunidades de alevinos de peixes encontrados em um dos pontos do Riacho 3, é um sinal positivo, pois estes grupos são sensíveis a variações ambientas, principalmente em $\mathrm{pH}$ e oxigênio dissolvido, dessa forma, sua presença sugere que o ambiente se encontra em processo inicial de degradação ambiental, tendo em vista a intervenção antrópica na comunidade é bem recente.

A presença, quantidade e distribuição de espécies indicadoras demonstram a intensidade dos impactos ambientais em um ecossistema aquático, podendo ser espécies, grupos ou comunidades biológicas (CALLISTO; GONÇALVES, 2002). As libélulas são insetos predadores que habitam os ecossistemas de água doce e estão associadas às plantas aquáticas e ao fundo dos corpos d'água, comportando-se como bioindicadores da qualidade ambiental, principalmente para o monitoramento de bacias hidrográficas (MARCO, 1998; ROMERO, 1998).

A presença de $M$. flexuosa (buriti), indica um ambiente relativamente descontaminado, uma vez que, essa espécie vegetal possui altos índices de taninos, que possuem carácter antimicrobiano relatado na literatura. As águas em torno de buritizais são escuras devido a quantidade de corantes naturais desta espécie, em contra partida, costumam ser translúcidas e livres de patógenos em excesso, proporcionando um ambiente com altos níveis de oxigenação dissolvida (MARTINI; MORUZZI, 2013; SOUSA, 2015).

\section{CONCLUSÕES}

Os parâmetros físico-químico e os dados qualitativos analisados sugerem que o Riacho 3 se encontra em processo inicial de degradação ambiental, provocado pelas recentes intervenções antrópicas no ambiente.

Esta análise quali-quantitativa da água do Riacho 3, emite um alerta aos poderes públicos municipais e estaduais, para tomada de medidas que visem a prevenção de maiores danos às águas. Dessa forma, são necessárias diligências tanto do poder público quanto dos ribeirinhos, para a conservação e uso racional destes recursos. Campanhas de sensibilização e conscientização com a comunidade são de extrema urgência, afim de reduzir o processo de degradação e antropização.

\section{REFERÊNCIAS}

ALVES, T. L. B.; LIMA, V. L. A.; FARIAS, A. A. Impactos ambientais no rio Paraíba na área do município de Caraúbas-PB: região contemplada pela integração com a bacia hidrográfica do Rio são Francisco. Revista Caminhos de Geografia, v. 13, n. 43, p. p. 160-173, 2012.

ARAÚJO, B. G. P.; OLIVA JÚNIOR, E. F. Abordagem dos Impactos Socioambientais no Riacho Limeira - Riachão 
do Dantas/SE. Revista Eletrônica da Faculdade José Augusto Vieira, v. 5, n 7, p. 16, 2012.

ARAÚJO, F. A. S. Geomorfologia aplicada à fragilidade e ao zoneamento ambiental de Caxias/MA. p. 184. Tese (doutorado) - Universidade Estadual Paulista Júlio de Mesquita Filho, Faculdade de Ciências e Tecnologia. 2012.

BARROS, M. C. Biodiversidade na Área de Proteção Ambiental Municipal do Inhamum. São Luís: UEMA, p. 142, 2012.

BRASIL. Código Florestal. Lei $n^{\circ} 4.471$ de 15 de setembro de $1965 . \quad$ Disponível em: https://www2.camara.leg.br/legin/fed/lei/1960-1969/lei4771-15-setembro-1965-369026-publicacaooriginal-1pl.html Acesso em: 10/05/2019.

BRASIL. Decreto 24.643 do Código das Águas (1934). Ministerio da Agricultura. Disponível em: http://www.planalto.gov.br/ccivil_03/decreto/d24643.ht m Acesso em: 23/08/2017.

BRASIL. Ministério do Meio Ambiente. Resolução CONAMA $\mathrm{n}^{\circ}$ 357, de 17 de Março de 2005. Publicada no DOU no 053, de 18/03/2005, págs. 58-63. Diário Oficial da União (retificado), Brasília-DF, 2005. Disponível em: http://www2.mma.gov.br/port/conama/legiabre.cfm?co dlegi=459 Acesso em: 23/08/2017.

BRASIL. Ministério do Meio Ambiente. Resolução CONAMA no 001 , de 23 de janeiro de 1986. Dispõe sobre critérios básicos e diretrizes gerais para a avaliação de impacto ambiental. Diário Oficial da União (retificado), Brasília-DF, 1986. Disponível em: < http://www.mma.gov.br/port/conama/processos/61AA3 835/LivroConama.pdf>. Acesso em: 23/08/2017.

CALLISTO, M.; GONCALVES, J. A vida nas águas das montanhas. Ciência Hoje, v. 31, n. 182, p. 68-71, 2002.

CARDOSO, R. S.; NOVAES, C. P. Variáveis Limnológicas e Macroinvertebrados Bentônicos como Bioindicadores de Qualidade da Água. Revista Nacional de Gerenciamento de Cidades, v. 1, n. 05, p. 16-35. 2013.

CONCEIÇÃO; G. M. RUGGIERI, A. C. Ocorrência e importância de Hidrolea spinosa L. (Hidrolaphyllaceae), Caxias, Maranhão-Brasil. Revista Acta Tecnológica, v. 5, n.1, p.4454, 2010.

COSTA, C. F.; AZEVEDO, C. A. S.; FERREIRA, S. S.; MOURA, E. P.

$S$. Análise microbiológica da água do Rio Itapecuru em Caxias-MA, Brasil. Revista Interface (Porto Nacional), $\mathrm{n}$. 10, p. 274-283.2016.

CREMONEZ, F. E.; CREMONEZ, P. A.; FEROLDI, M.; CAMARGO, M. P.; KLAJN, F. F.; FEIDEN, A. Avaliação de impacto ambiental: metodologias aplicadas no Brasil. Monografias Ambientais, v. 13, n. 5, p. 3821-3830, 2014.

ESTEVES, F. D. A. Fundamentos de Limnologia. Interciência,
Rio de Janeiro, n. 2ª , p.226, 1998

GASPAR, J. C.; SILVA, G. S.; SILVA, M. L. A.; SILVA, W. F. N. CONCEIÇÃO, G. M. Degradações Ambientais no Riacho Ouro em Trechos da Zona Rural do Município de Caxias, Maranhão. Agrarian Academy. v. 3, n. 6, p. 150, 2016.

IBGE. Censo Demográfico. Instituto Brasileiro de Geografia e Estatística-IBGE, 2010. Disponível em: www.ibge.gov.br. Acesso em: 23/08/2017.

LEMOS, M.; NETO, M. F.; DIAS, N. A. Sazonalidade e variabilidade espacial da qualidade da água na Lagoa do Apodi, RN. Revista Brasileira de Engenharia Agrícola e Ambiental, v. 14, n. 2, p.155-164, 2010.

MARCO, P. The Amazonian Campina dragonfly assemblage: patterns in microhabitat use and behaviour in a foraging habitat (Anisoptera). Odonatologica, v. 27, n. 2, p. 239248, 1998.

MARTINI, M. V. P.; MORUZZI, R. B. Tratabilidade de águas pluviais utilizando coagulante natural a base de tanino visando fins não potáveis. Teoria e Prática na Engenharia Civil. v. 13, n. 22, p. 15-23, 2013.

MATSUMURA-TUNDISI, T., TUNDISI, J. G. Limnologia. Oficina de Textos, São Paulo. p. 632, 2008.

NOGUEIRA, F. F.; COSTA, I. A.; PEREIRA, U. A. Análise de parâmetros físico-químicos da água e do uso e ocupação do solo na sub-bacia do Córrego da Água Branca no município de Nerópolis-Goiás. Universidade Federal de Goiás. Escola de Engenharia Civil e Ambiental. Curso de Engenharia Ambiental e Sanitária. p. 53, 2015.

OLIVEIRA, R. M. M.; SANTOS, E. V.; LIMA, K. C. Avalição da Qualidade do Riacho São Caetano, de Balsas, MA, com base em Parâmetro Fisico, Químicos e Microbiológicos. Revista de Engenharia Sanitária e Ambiental. v. 22, n. 3, p. 523-530, 2017.

RIBEIRO, A. H. O Buriti (Mauritia flexuosa L. f.) na Terra Indígena Araçá, Roraima: usos tradicionais, manejo e potencial produtivo. p. 101. Dissertação (Mestrado em Manejo florestal, Silvicultura) - Instituto Nacional de Pesquisas da Amazônia, Manaus, 2010.

ROMERO, M. F.. New data on the ecological tolerance of some rheophilous Odonata in Mediterranean Europe (Sierra Morena, Southern Spain). Odonatologica, v. 17, n. 2, p. 121-126, 1988.

SILVA, D. J.; CONCEIÇÃO, G. M. Rio Itapecuru: Caracterização Geoambiental e Socioambiental, Município de Caxias, Maranhão, Brasil. Scientia Plena, v. 7, n. 1, p. 26, 2011.

SILVA, P. A. Orthopsittaca manilata (Boddaert, 1783) (Avian: Psittacidae): abundance and feeding activity in relation to Mauritia flexuosa (Arecaceae) palm swamp fructification in Triângulo Mineiro. p. 71. Dissertação (Mestrado em Ciências Biológicas) - Universidade Federal de Uberlândia, Uberlândia, 2009. 
André Ricardo Ferreira da Silva Rocha, Helenice Silva Gomes, Ricardo Clayton Silva Jansen, Tatiane Neves de Sousa, Domingas Hosanira Silva de Sousa, Gustavo da Silva Gomes, Guilherme Sousa da Silva, Jailson da Costa Gaspar, Maria de Fátima Veras Araujo, Gonçalo Mendes da Conceição

SILVA, T. S. S. Estudo de trailidade físico-química com uso de taninos vegetais em água de abastecimento e esgoto. p. 83. Tese de Doutorado. Fundação Oswaldo Cruz. Escola Nacional de Saúde Pública. Pós Graduação de Saúde Pública. 1999.

SOUSA, T. B. Uso de taninos de espécies florestais no tratamento de água para abastecimento. p. 97. Dissertação de Mestrado. Universidade Federal de Lavras. Programa de Pós-Graduação em Ciência e Tecnologia da Madeira. 2015.

USA FEDERATION. Water Environmental et al. Standard Methods for the Examination of Water and Wastewater. American Public Health Association (APHA): Washington, DC, USA, p. 440, 2012.

VON SPERLING, M. Estudos e modelagem da qualidade da água de rios. Príncipios do tratamento biológico de águas residuárias. 3ed. Belo Horizonte: UFMG, v. 7 p. 588, 2007.

WWAP (United Nations World Water Assessment Programme). The United Nations World Water Development Report 2017: Wastewater, The Untapped Resource. Paris, UNESCO. p. 313, 2017. 